\title{
УДК 513.881
}

\section{О БАЗИСАХ В ПРОСТРАНСТВАХ НЕПРЕРЫВНЫХ n-ОДНОРОДНЫХ ПОЛИНОМОВ, ДЕЙСТВУЮЩИХ В ЯДЕРНЫХ ПРОСТРАНСТВАХ КЁТЕ}

\author{
В. П. Кондаков
}

В работе строится базис в пространстве непрерывных $n$-однородных полиномов, отображающих ядерное пространство Кёте - Фреше в себя. Пространства полиномов рассматриваются с топологиями равномерной сходимости на ограниченных множествах пространств Кёте. Обсуждаются и близкие вопросы.

Ключевые слова: голоморфное отображение, $n$-однородный полином, базис.

Цель настоящей статьи - построение базисов в пространствах непрерывных $n$-однородных полиномов и голоморфных функций, отображающих ядерные пространства Кёте - Фреше в себя, с топологиями равномерной сходимости на всех ограниченных (предкомпактных) множествах.

Частный случай при $n=1$ (базис в пространстве непрерывных линейных операторов) изложен, например, в [1]. Базис, аналогичный классическому степенному базису, в пространстве голоморфных $\mathbb{C}$-значных функций на пространствах из некоторого класса ядерных пространств рассмотрен в [2] (см. также [3]).

\section{1. Предварительные сведения}

Напомним необходимые общие определения и факты, которые более подробно изложены в $[3,4]$.

Пусть $E, F-$ линейные пространства над полем комплексных чисел. Пространство $n$-линейных отображений из $E$ в $F$ будем обозначать $\mathscr{L}\left({ }^{n} E, F\right), n \in \mathbb{N}$. Если $L \in \mathscr{L}\left({ }^{n} E, F\right)$, то $L$ определено на $E^{n}$ (декартовом произведении $n$-экземпляров пространства $E$ ) со значениями в $F$ и оно линейно по каждому переменному при фиксированных остальных.

ОПРеДЕЛЕНИЕ 1. $n$-линейное отображение $L$ из $E$ в $F$ называют симметричным, если $L\left(x_{1}, \ldots, x_{n}\right)=L\left(x_{\sigma(1)}, \ldots, x_{\sigma(1)}\right)$ для любого набора элементов $x_{1}, \ldots, x_{n} \in E$ и любой перестановки $\sigma$ первых $n$ натуральных чисел.

Пусть $\Delta$ обозначает диагональное отображение из $E$ в $E^{n}$, которое действует по правилу

$$
\Delta(x)=(x, \ldots, x) \in E^{n} \quad(\forall x \in E) .
$$

ОПРЕДЕЛЕНИЕ 2. Композицию диагонального отображения $\Delta: E \rightarrow E^{n}$ и $n$-линейного отображения $L_{n}$, определенного на $E$, со значениями в $F$ называют $n$-однородным

(c) 2012 Кондаков В. П. 
полиномом, отображающим локально выпуклое пространство $E$ в локально выпуклое пространство $F$. Пространство всех $n$-однородных полиномов из $E$ в $F$ обозначают $\mathscr{P}\left({ }^{n} E, F\right)$.

Таким образом, если $P \in \mathscr{P}\left({ }^{n} E, F\right)$, то $P=L_{n} \circ \Delta$, где $L_{n}-n$-линейное отображение.

Описанное представление $P$ не является единственным. Оно становится единственным, если потребовать симметричность $n$-линейной формы $L_{n}$ (подробнее см., например, [3]). В последнем случае $L_{n}$ называют полярной формой, определяемой $n$-однородным полиномом $P$. Значения полярной формы $L_{n}$ определяются значениями $P$ по поляризационной формуле

$$
L_{n}\left(x_{1}, \ldots, x_{n}\right)=\frac{1}{2^{n} n !} \sum_{\varepsilon_{i}= \pm 1} \varepsilon_{1} \ldots \varepsilon_{n} P\left(\sum_{k=1}^{n} \varepsilon_{k} x_{k}\right) .
$$

Пусть $E$ - локально выпуклое, а $F$ - счетнонормированное пространства. Везде ниже в пространстве непрерывных отображений $f$ из $E$ в $F$ рассматривается топология равномерной сходимости на всех ограниченных множествах из $E$. Эта топология задается системой непрерывных полунорм, каждая из которых определяется ограниченным множеством $A \subset E$ и индексом $r \in \mathbb{N}$ счетного набора полунорм $(\|\cdot\|)_{r \in \mathbb{N}}$ (задающего топологию $F$ ) следующим образом:

$$
\|f\|_{A, r}=\sup _{x \in A}\|f(x)\|_{r} .
$$

Каждой такой полунорме соответствует полунорма в пространстве $n$-линейных отображений, действующих из $E^{n}$ в $F$, вида

$$
\|L\|_{A^{n}, r}=\sup _{\left(x_{1}, \ldots, x_{n}\right) \in A^{n}}=\left\|L\left(x_{1}, \ldots, x_{n}\right)\right\|_{r}, \text { где } A^{n}=A \times A \times \ldots \times A \subset E^{n} .
$$

Приведем определение пространств Кёте - Фреше, на которых будут рассматриваться ниже $n$-однородные полиномы и голоморфные функции.

Пусть $\left[a_{r}(n)\right]_{r, n \in \mathbb{N}}$ - матрица неотрицательных чисел со свойством монотонности по строкам, определяемым индексом $r \in \mathbb{N}$,

$$
0 \leqslant a_{r}(n) \leqslant a_{r+1}(n) \leqslant \ldots \quad(\forall r, n \in \mathbb{N})
$$

- матрица Кёте.

Пространством Кёте - Фреше называют пространство последовательностей комплексных чисел

$$
l_{p}\left[a_{r}(n)\right]=\left\{\xi=\left(\xi_{n}\right)_{n=1}^{\infty}:\left(\sum_{n=1}^{\infty}\left|\xi_{n}\right|^{p} a_{r}^{p}(n)\right)^{\frac{1}{p}} \doteq\|\xi\|_{r}<+\infty \quad \forall r\right\} \quad(1 \leqslant p \leqslant \infty)
$$

с топологией, задаваемой системой полунорм $\left(\|\cdot\|_{r}\right)_{n=1}^{\infty}$. Каждый элемент пространства Кёте - Фреше имеет единственное представление по базису ортов $\left(e_{n}\right)$ :

$$
\xi=\sum_{n=1}^{\infty} e_{n}^{\prime}(\xi) e_{n}=\sum_{n=1}^{\infty} \xi_{n} e_{n} \quad\left(\left\|e_{n}\right\|_{r}=a_{r}(n)\right)
$$

в виде сходящегося ряда, где $e_{n}^{\prime}(\cdot)$ - координатные функционалы, составляющие базис сильного сопряженного $E_{\beta}^{\prime}$ к $E=l_{p}\left[a_{r}(n)\right]$. 
Известное условие ядерности пространства Кёте - Фреше состоит в том, что

$$
(\forall r)(\exists s(r)) \quad \sum_{n=1}^{\infty} \frac{\left\|e_{n}\right\|_{r}}{\left\|e_{n}\right\|_{s(r)}}=\sum_{n=1}^{\infty} \frac{a_{r}(n)}{a_{s(r)}(n)}<+\infty .
$$

Сильное сопряженное $E_{\beta}^{\prime}$ к ядерному пространству Кёте - Фреше также ядерно (см., например, [5]), и ниже будет использовано соответствующее условие его ядерности в терминах полунорм базиса $\left(e_{n}^{\prime}(\cdot)\right)_{n=1}^{\infty}$. Нам потребуется также определение голоморфного отображения (см., например, [6]).

Пусть $\mathscr{P}\left({ }^{n} E, F\right)$ при любом $n$ наделены топологией равномерной сходимости на всех ограниченных множествах из $E$.

ОПреДЕЛЕниЕ 3. Отображение $f: E \rightarrow F$ называется голоморфным, если существует последовательность $P_{n} \in \mathscr{P}\left({ }^{n} E, F\right)$ такая, что ряды $\sum_{n=0}^{\infty} P_{n}(x)$ сходятся к $f(x)$ для каждого $x \in X$.

Будем рассматривать пространство голоморфных отображений из $E$ в $F$, которые еще и ограничены на каждом ограниченном подмножестве из $E$. Это пространство обозначим $\mathscr{H}_{b}(E, F)$ и будем говорить, что элементы этого пространства есть голоморфные отображения ограниченного типа.

\section{2. Основные результаты}

Теорема 1. Пусть $E=l_{2}\left[a_{r}(n)\right]$ - ядерное пространство Кёте - Фреше. Пространство непрерывных $n$-однородных полиномов $P\left({ }^{n} E, E\right)$ с топологией равномерной сходимости на ограниченных множествах в $Е$ является ядерным пространством с абсолютным базисом

$$
\left\{T_{i j}(\cdot)=e_{i_{1}}^{\prime}(\cdot) e_{i_{2}}^{\prime}(\cdot) \ldots e_{i_{n}}^{\prime}(\cdot) e_{j}, j \in \mathbb{N}, i_{k} \in \mathbb{N}, k=1,2, \ldots, n\right\} .
$$

$\triangleleft$ Любой оператор - n-однородный полином $P \in P\left({ }^{n} E, E\right)$ допускает формальное разложение по системе $\left\{T_{i j}\right\}$

$$
\begin{gathered}
P e=L_{n}(e, e, \ldots, e)=L_{n}\left(\sum_{i=1}^{\infty} e_{i}^{\prime}(e) e_{i}, \ldots, \sum_{i=1}^{\infty} e_{i}^{\prime}(e) e_{i}\right) \\
=\sum_{i_{1}, \ldots, i_{n}=1}^{\infty} e_{i_{1}}^{\prime}(e) \ldots e_{i_{n}}^{\prime}(e) L_{n}\left(e_{i_{1}}, e_{i_{2}}, \ldots, e_{i_{n}}\right) \\
=\sum_{i_{1}, \ldots, i_{n}=1}^{\infty} \sum_{j=1}^{\infty} e_{j}^{\prime}\left(L_{n}\left(e_{i_{1}}, e_{i_{2}}, \ldots, e_{i_{n}}\right)\right) e_{i_{1}}^{\prime}(e) \cdot \ldots \cdot e_{i_{n}}^{\prime}(e) e_{j},
\end{gathered}
$$

где $L_{n}$ - симметрическая $n$-линейная форма, соответствующая $n$-однородному полиномy $P$.

Многомерную бесконечную матрицу $\left(e_{j}^{\prime}\left(L_{n}\left(e_{i_{1}}, e_{i_{2}}, \ldots, e_{i_{n}}\right)\right)\right)$ можно рассматривать как обобщение матрицы конечномерного оператора.

Пусть теперь $A$ - ограниченное множество в $E$, а $r$ - натуральный индекс окрестности нуля $V_{r}$ счетнонормированного пространства $\left(F,\left(V_{k}\right)_{k=1}^{\infty}\right)$. Если $A$ пробегает совокупность всех ограниченных множеств в $E$ и, независимо, $r$ - натуральный ряд, то система полунорм

$$
\left\{\|P\|_{A, r}=\sup _{e \in A}\|P e\|_{r}\right\}
$$

задает топологию равномерной сходимости на всех ограниченных множествах из $E$ в $P\left({ }^{n} E, E\right)$. 
Из поляризационной формулы следует простая известная оценка

$$
\left\|L_{n}\right\|_{A^{n}, r} \leqslant \frac{n^{n}}{n !}\|P\|_{A, r}
$$

для любого непрерывного $n$-однородного полинома $P$ и определяемой им полярной формы $L_{n}$ (подробнее см., например, [3, теорема 1.7]).

С использованием формулы Стирлинга из последней оценки непосредственно выводится необходимая нам оценка следующей леммы.

Лемма. Пусть $P=L_{n} \circ \Delta-$ непрерывный $n$-однородный полином, отображающий локально выпуклое пространство $E$ в счетнонормированное пространство $F$ с системой полунорм $\left(\|\cdot\|_{r}\right)_{r \in \mathbb{N}}$. Тогда для любого ограниченного множества $A \subset E$ и натурального $r$ c некоторой константой $c$ (не зависящей от $P, n, A, r)$ справедлива оценка

$$
\left\|L_{n}\right\|_{A^{n}, r} \leqslant c e^{n}\|P\|_{A, r} .
$$

В пространстве Кёте - Фреше базис замкнутых абсолютно выпуклых ограниченных множеств (насыщенное семейство) можно выбирать состоящим из $p$-эллипсоидов, оси которых имеют направления элементов базиса ортов (см., например, [1]). В этом случае полунормы элементов $e_{n}^{\prime}$ в $E_{\beta}^{\prime}$ вычисляются аналогично вычислению норм координатных функционалов базиса ортов в банаховых пространствах $l_{p}(1 \leqslant p \leqslant \infty)$, которые также причисляются к пространствам Кёте (случай $\left.a_{r}(n) \equiv 1\right)$. А именно, если $C-$ ограниченное множество (

$$
\left\|e_{k}^{\prime}\right\|_{C^{\circ}}=\sup _{e \in C}\left|e_{k}^{\prime}(e)\right|=\left\|e_{k}\right\|_{C}^{-1}, \text { где }\left\|e_{k}\right\|_{C}=\inf \left\{\lambda>0: e_{k} \in \lambda C\right\} .
$$

Тогда, обозначив символом $\mathscr{A}-$ базис ограниченных множеств, состоящий из $p$-эллипсоидов, будем иметь условие ядерности сильного сопряженного $E_{\beta}^{\prime}$

$$
(\forall A \in \mathscr{A})(\exists B \in \mathscr{A}) \quad \sum k=1^{\infty} \frac{\left\|e_{k}^{\prime}\right\|_{A^{\circ}}^{\prime}}{\left\|e_{k}\right\|_{B^{\circ}}^{\prime}}=\sum_{n=1}^{\infty} \frac{\left\|e_{k}\right\|_{B}}{\left\|e_{k}\right\|_{A}}<+\infty .
$$

Заметим, что в [1] показана возможность выбора системы ограниченных $p$-эллипсоидов, при котором $\left\|e_{k}\right\|_{A}=\left\|e_{k}\right\|_{r(k)}$ при некоторой (зависящей от $A$ ) последовательности $r(k) \rightarrow \infty$. Тогда последнее условие ядерности $E_{\beta}^{\prime}$ выводится непосредственно из условия ядерности пространства Кёте - Фреше $E$.

Рассмотрим на $P\left({ }^{n} E, E\right)$ новую систему полунорм

$$
\left\{\|P\|_{A, r}=\sum_{\substack{j, i_{k}=1, k \leqslant n}}^{\infty}\left|e_{j}^{\prime}\left(L_{n}\left(e_{i_{1}}, \ldots, e_{i_{n}}\right)\right)\right|\left\|e_{i_{1}}^{\prime}(\cdot) \ldots e_{i_{n}}^{\prime}(\cdot) e_{j}\right\|_{A, r}, A \in \mathscr{A}, r \in \mathbb{N}\right\},
$$

задающую топологию, которая в силу неравенства треугольника не слабее исходной (в $\left.\left(P\left({ }^{n} E, E\right),\left\{\|\cdot\|_{A, r}\right\}\right)\right)$.

Покажем, что новая система полунорм эквивалентна исходной.

С использованием условий ядерности, непрерывности $P$ (а значит и $\left.L_{n}\right)$ и леммы 
имеем

$$
\begin{gathered}
\|P\|_{A, r} \leqslant\|P\|_{A, r} \leqslant \sum_{\substack{j, i_{k}=1, k \leqslant n}}^{\infty}\left\|e_{j}^{\prime}\right\|_{s(r)}^{\prime}\left\|L_{n}\left(e_{i_{1}}, e_{i_{2}}, \ldots, e_{i_{n}}\right)\right\|\left\|T_{i j}\right\|_{A, r} \\
\leqslant \sum_{\substack{j, i_{k}=1, k \leqslant n}}^{\infty} \frac{1}{\left\|e_{j}\right\|_{s(r)}}\left\|L_{n}\right\|_{s_{1}(r)} \prod_{k=1}^{n}\left\|e_{i_{k}}\right\|_{B} \prod_{k=1}^{n}\left\|e_{i_{k}}^{\prime}\right\|_{A^{\circ}}^{\prime}\left\|e_{j}\right\|_{r} \\
\leqslant\left\|L_{n}\right\|_{B, s_{1}(r)} \sum_{\substack{j, i_{k}=1, k \leqslant n}}^{\infty} \frac{\left\|e_{j}\right\|_{r}}{\left\|e_{j}\right\|_{s(r)}} \prod_{k=1}^{n} \frac{\left\|e_{i_{k}}\right\|_{B}}{\left\|e_{i_{k}}\right\|_{A}} \leqslant C\|P\|_{B, s_{1}(r)} \cdot \triangleright
\end{gathered}
$$

Теорема 2. В пространстве непрерывных голоморфных отображений $T: E \rightarrow E$, где $E$ - ядерное пространство Кёте - Фреше, наделенном топологией равномерной сходимости на всех ограниченных множествах из $E$, система одномерных операторов

$$
\left\{T_{i j}(\cdot)=\prod_{k=1}^{n} e_{i_{k}}^{\prime}(\cdot) e_{j}, \quad j \in \mathbb{N}, i_{k} \in \mathbb{N}, k \leqslant n, n=1,2, \ldots\right\}
$$

образует абсолютный равностепенно непрерывный базис.

Доказательство может быть проведено с использованием утверждения теоремы 1 и исследованного многими авторами характера разложения голоморфной функции в ряд по $n$-однородным полиномам (аналог разложения в ряд Тэйлора), но есть и другой путь установить абсолютность и равностепенную непрерывность системы $\left(T_{i j}\right)$, который представляется более прямым и дословно повторяет оценки работы [2]. Для этого необходимо перегруппировать формальное разложение функции $f$ и получить аналог степенного ряда

$$
f(x)=\sum_{m \in \mathbb{N}^{\mathbb{N}}} a_{m} x^{m}
$$

где $\left.a_{m} \in E, x^{m}=\left(e_{i_{1}}^{\prime}(x)\right)^{m_{1}} \cdot \ldots \cdot e_{i_{k}}^{\prime}(x)\right)^{m_{k}}, \mathbb{N}^{\mathbb{N}}-$ множество наборов натуральных чисел $m=\left(m_{1}, \ldots m_{k}, \ldots\right), m_{j}=0$ для всех $j>j(m)$.

Решающим моментом является то, что в качестве ограниченных множеств $A$ и $B$ можно брать упомянутые выше соосные эллипсоиды, ряд из отношений полуосей которых сходится (точнее, для эллипсоида $A$ найдется ограниченный эллипсоид $B$ с большими полуосями). Отличие от рассмотренного в [2] случая $a_{m}$, модули которых оцениваются с использованием формулы Коши, в конце концов разлагаются по базису $\left(e_{j}\right)$ и это завершает доказательство.

Следствие. В пространстве голоморфных отображений, описанном в теореме 2 , подпространство непрерывных $n$-однородных полиномов является дополняемым при любом $n$.

Анализ приведенных и упомянутых оценок из работ $[2,3,6-10]$, вероятно, позволит обобщить и частично распространить полученные в теоремах 1-2 утверждения на случай пространств голоморфных отображений из $E$ в $F$, где $E$ и $F$ - пространства из более широких классов, например, класса совершенно ядерных пространств с базисом Шаудера (см. определение в [2, 3]).

Заметим, что необходимость условия ядерности пространств для абсолютности рассматриваемых базисов следует из результата работы [11]. 


\section{Литература}

1. Кондаков В. П. Три основных принципа линейного функционального анализа, их обобщения и приложения.-Владикавказ: ВНЦ РАН, 2007.-208 с.

2. Boland P. J., Dineen S. Holomorphic functions on fully nuclear spaces // Bull. Soc. Math. France.1978.-Vol. 106.-P. 311-336.

3. Dineen S. Complex analysis in locally convex spaces // Math. Stud.-North-Holland, 1981.-Vol. 57.

4. Hille E., Phillips R. S. Functional Analysis and Semi-Groups // Amer. Math. Soc. Colloq. Pub.Providence (R. I.): Amer. Math. Soc., 1957.-Vol. 31.-P. 796.

5. Пич А. Ядерные локально выпуклые пространства.-М.: Мир, 1967.-266 с.

6. Ryan R. A. Holomorphic mappings on $l_{1} / /$ Trans. Amer. Math. Soc.-1987.-Vol. 302.-P. 797-811.

7. Dineen $S$. Analytic functionals on fully nuclear spaces // Stud. Math.-1982.-Vol. 73.-P. 11-32.

8. Хренников А. Ю., Петерсон Х. Теорема Пэли - Винера для обобщенных целых функций на бесконечномерных пространствах // Изв. РАН. Сер. мат.-2001.-Т. 65, вып. 2.-С. 201-224.

9. Кондаков В. П. О представлении в виде пространств Кёте пространств голоморфных функций // Владикавк. мат. журн.-2005.-Т. 7, вып. 4.-С. 22-29.

10. Кондаков В. П. О дифференцируемости отображений и строении пространств голоморфных функций на пространствах числовых последовательностей // Владикавк. мат. журн.-2007.-Т. 9, вып. 2.-C. 9-21.

11. Dineen S., Timoney R. M. Absolute bases, tensor products and a theorem of Bohr // Stud. Math.1989.--Vol. 94.-P. 227-234.

Статъя поступила 5 июля 2011 г.

КОНДАКОВ ВЛАДИМИР ПЕТРОВИч

Южный федеральный университет,

заведующий каф. теории функций

и функционального анализа

РОССИЯ, 344090, Ростов-на-Дону, ул. Мильчакова, 8 a;

Южный математический институт ВНЦ РАН и РСО-А,

заведующий лабораторией вещественного анализа

РОССИЯ, 362027, Владикавказ, ул. Маркуса, 22

E-mail: kond@math.rsu.ru

\section{ON BASES IN SPACES OF CONTINUOUS $n$-HOMOGENEOUS POLYNOMIALS IN NUCLEAR KÖTHE SPACES}

\section{Kondakov V. P.}

A basis in the space of continuous $n$-homogeneous polynomials mappings a nuclear Köthe - Frechet space into itself is constructed. The space of polynomials is considered with the compact open topology. Some related problems are also discussed.

Key words: holomorphie mapping, $n$-homogeneous polynomial, basis. 